\title{
Enhancement of the accuracy of single epoch GPS positioning for long baselines by local ionospheric modelling
}

\author{
M. Assiadi* · S. J. Edwards · P. J. Clarke \\ School of Civil Engineering and Geosciences, Newcastle University, Newcastle upon Tyne NE1 7RU, United \\ Kingdom \\ * corresponding author, tel +44(0)191222 6445, fax +44 (0)191222 6502, e-mail \\ Mohammed.Assiadi@newcastle.ac.uk
}

This is an author preprint of a paper accepted by GPS Solutions. The final publication is available at Springer via doi:10.1007/s10291-013-0344-6

\begin{abstract}
Single-epoch relative GPS positioning has many advantages, especially for monitoring dynamic targets .In this technique, errors occurring in previous epochs cannot affect the position accuracy at the current epoch, but careful processing is required, and resolving carrier phase ambiguities is essential. Statistical ambiguity resolution functions have been used to determine the best values of these ambiguities. The function inputs include as a minimum the known base station position, the approximate roving antenna 'seed' position, and the dual-frequency carrier phase measurements from both receivers. We investigate different solutions to find the ambiguity function inputs that achieve the highest ambiguity resolution success rate. First, we address the rover seed position. A regionally-filtered undifferenced pseudorange coordinate solution proves better than a double-differenced one. Multipath errors approximately repeat themselves every sidereal day in the case of static or quasi-static antennas; applying a sidereal filter to the pseudorange-derived positions mitigates their effects. Second, we address the relative carrier phase measurements, which for medium to long baselines are significantly affected by ionospheric propagation errors imperfectly removed during differencing. In addition to the IGS ionospheric model, we generate a local pseudorange-based ionospheric correction. Applying this correction improves the quality of the phase measurements, leading to more successful ambiguity resolution. Temporally smoothing the correction by means of a Kalman filter further improves the phase measurements. For baselines in the range $60-120 \mathrm{~km}$, the mean absolute deviation of single-epoch coordinates improves to $10-20 \mathrm{~cm}$, from $30-50 \mathrm{~cm}$ in the default case.
\end{abstract}

Keywords GPS, Single-epoch positioning, Ambiguity resolution, Sidereal filter, Ionosphere, Kalman filter.

\section{Introduction}

Using single-epoch GPS positioning has many advantages in high-multipath and restricted sky visibility situations, especially when monitoring dynamic targets where sudden and unpredictable movements occur. In this technique, each data epoch is processed independently and so measurement errors and outages occurring in previous epochs cannot affect the current epoch's accuracy. However, double-differenced GPS carrier phase measurements are biased by an unknown integer number of cycles, often called the double-difference 
ambiguity. Resolving this ambiguity efficiently and correctly remains one of the greatest challenges, especially for epoch by epoch GPS applications where the number of observations is limited by the number of observed satellites at each epoch. A variety of ambiguity resolution functions has been introduced (Kim and Langley 2000). These functions attempt to resolve the ambiguities for each epoch of processed data separately. On occasion there may not be any redundancy in the observations, and then the goodness of fit of the ambiguity resolution cannot be tested. As a result, strict attention must be paid to the other positioning errors such as receiver dependent biases, satellite dependent biases and signal propagation biases i.e. ionospheric and tropospheric delays. These errors must be eliminated to ensure correct ambiguity resolution and thus obtain the highest possible accuracy.

For relative GPS over medium to long baselines of $30-100 \mathrm{~km}$, the residual error budget is dominated by ionospheric propagation errors (Lejeune et al. 2012). We develop a local code pseudorange-based ionospheric zenith delay correction model to augment the IGS 2D ionospheric model. These models are used to provide improved a priori roving antenna coordinates and corrected double-difference carrier phase observations. We examine different methods of derivation of the local model, and the effects of these ionospheric models and of regional and sidereal position filtering techniques on the success of positioning. Finally, we examine the use of Kalman filtering to reduce noise in the local ionospheric model by temporal smoothing.

\section{Positioning algorithm and choice of initial coordinates}

This research uses the GPS Ambiguity Search Program (GASP) single-epoch software developed at Newcastle University (Corbett and Cross 1995). GASP uses the Ambiguity Function Method (AFM) for resolving the double differenced phase integer ambiguities (Counselman and Gourevitch 1981; Mader 1992). The AFM relies on the assumption that all the errors that affect the GPS carrier phase are mitigated, so the double-differenced phase observation equation is of the form

$$
\varphi_{i}^{j}(t)=\frac{f_{i}}{c} \rho^{j}(t)+N_{i}^{j}
$$

Here, $\varphi_{i}^{j}(t)$ is the carrier phase double-difference observation on frequency $i$ from two receivers and satellite $j$ relative to an arbitrary reference satellite, $\rho^{j}$ is the computed or estimated double-difference geometric distance, $N_{i}^{j}$ is the double-difference initial integer ambiguity, $c$ is the speed of light, and $f_{i}$ is the signal frequency.

The AFM search volume is formed around the roving antenna's initial seed position, which may be specified a priori for a static or slow-moving antenna, or computed using the GPS pseudorange observations in the case of a highly dynamic antenna. Within the search volume, candidate positions are tested using the 
Ambiguity Function Value (AFV) statistic. For a single epoch of data spanning a baseline, the AFV at a test rover position $(x, y, z)$ can be represented as follows (Counselman and Gourevitch 1981):

$$
\operatorname{AFV}(x, y, z)=\frac{1}{n_{f}\left(n_{s}-1\right)} \sum_{1=1}^{n_{s}-1} \sum_{i=1}^{n_{f}} \cos 2 \pi\left(\varphi_{i}^{j}-\frac{f_{i}}{c} \rho^{j}\right)
$$

where $n_{f}$ is the number of observation frequencies, and $n_{s}$ is the number of observed satellites. In our case, $n_{f}=2$. Because the AFV depends on both GPS frequencies individually, the ionospheric effect should be eliminated before evaluating this function, or the baseline should be limited to a length less than $10 \mathrm{~km}$ for which the ionospheric errors are effectively removed when differencing is applied (Counselman and Gourevitch 1981).

The trial coordinates are found by adding increments to an initial antenna seed position. The creation of the search volume in GASP uses four chosen satellites, the highest elevation satellite as a reference and the other three satellites which together with the reference satellite give the best Positional Dilution of Precision (PDOP) geometry. The volume includes all the possible positions as the double-difference carrier phases are perturbed by integer numbers of cycles within a specified search range, in our case 5 cycles, surrounding the rounded values at the seed position. For the method to work, it is heuristically found that the seed coordinates should be within $\pm 1.2 \mathrm{~m}$ of the correct final position.

In contrast to the trial volume determination that only uses the four 'best' satellites as described above, the ambiguity function search for the best rover location involves L1 and L2 observations of all of the possible double differenced observations for all the satellites above the minimum allowed elevation angle. A statistical Ftest is performed on all of the successful candidate positions, which have an AFV greater than the specified threshold, to identify the final correct position for each epoch. The ratio of each candidate position's a posteriori residual variance to that of the minimum variance candidate position is tested at $5 \%$ significance. If another candidate position is indistinguishable from the minimum variance candidate, then the epoch is rejected.

Equation (1) assumes that all observational biases have been removed, which we attempt as described here. We use International GNSS Service (IGS) Final satellite orbital positions and clock data (Kouba 2009). Satellite and ground antenna phase centre offsets are removed from the undifferenced measurements using IGS values, as are elevation- and azimuth-dependent ground antenna phase centre variation corrections (Schmid et al. 2005). The undifferenced measurements are also corrected for the relativistic Sagnac effect (Leick 2004), for the ionospheric propagation error using the IGS single-layer global model and mapping function (Schaer et al. 1998), and for the hydrostatic neutral atmosphere delay using the Saastamoinen model and Niell mapping function (Saastamoinen 1972; Niell 1996). Inter-frequency code pseudorange biases are corrected using the calibration values associated with the IGS ionospheric model. Remaining atmospheric, receiver and satellite 
hardware and clock biases are negligible in the code pseudorange solution, and are largely cancelled in the carrier phase solution by applying the double differencing technique, as are solid earth tidal deformation effects.

In this research, different single epoch pseudorange solutions have been tested to find the best inputs for the GASP ambiguity function, namely the rover antenna seed position and the corrected GPS phase observations (2). These inputs may be obtained by solving double-differenced or undifferenced pseudorange observation equations (Table 1). Our base 'solution 1' is a relative single-frequency pseudorange solution where we solve for the rover coordinates and receiver clock term only (as described above, the IGS single layer ionospheric model is applied to all measurements before any differencing takes place). Second ('solution 2'), we additionally use the geometry-free undifferenced pseudorange observables at the base station and rover to solve for pseudorange-based ionospheric models on each satellite individually, to absorb the residual ionospheric effect that is not described by the coarse IGS model which has $2.5^{\circ}$ latitude and $5^{\circ}$ longitude resolution. Alternatively, solution 3 uses the pseudorange observation equations on both frequencies to solve directly for residual zenith ionospheric delay, simultaneously with the antenna position and clock, at the base and rover separately. Solution 4 augments solution 3 by applying a regional filter to the rover coordinates, based on the apparent displacement of the base station, to remove residual positioning errors in the pseudorange solution at the rover. Finally, solution 5 incorporates a sidereal position filter to absorb repeating errors in the rover position at the geometry repeat interval of $86154 \mathrm{~s}$ (Ragheb et al. 2007).

The five different processing strategies used in this research are summarised in Table 1. In this table, $f_{1}$ and $f_{2}$ are the two GPS signal frequencies on L1 and L2, $C_{1}, P_{1}$ and $P_{2}$ are pseudorange measurements on L1 and L2 frequencies, $\Phi_{1}$ and $\Phi_{2}$ are carrier phase measurements on L1 and L2 frequencies in metric units, $\Phi_{1 c}$ and $\Phi_{2 c}$ are their ionosphere-corrected equivalents, $\rho$ is the geometric distance between satellite and ground antennas, $c$ is the speed of light, and $d T$ is the receiver clock error in seconds, $I_{R}$ is the residual zenith ionosphere delay on the L1 frequency in metres, $\gamma_{i}$ is the unitless scale factor for converting ionosphere delay from L1 to frequency $i\left(77^{2} / 60^{2}\right.$ for L2), $M(e)$ is the ionospheric mapping function dependent on elevation only, $X_{m 0}$ and $X_{f 0}$ are the apparent instantaneous coordinate vectors of the rover and base station respectively, estimated from the pseudorange solution, $X_{f t}$ and $X_{m t}$ are the "true" (IGS) base station and rover position vectors, $X_{r}$ is the estimated regionally-filtered rover coordinate vector, $X_{r p}$ is its value on the previous mean sidereal day, and $X_{s}$ is the estimated regionally- and sidereally-filtered rover coordinate vector. Superscript $j$ in solutions 1 and 2 denotes double-difference measurements along a baseline for satellite $j$ with respect to a chosen reference satellite; all quantities without this subscript are undifferenced.

Table 1. Combinations of pseudorange observable used to generate seed positions, and residual ionospheric corrections to the carrier phase, used by the GASP ambiguity function 


\begin{tabular}{|c|c|c|c|}
\hline $\begin{array}{l}\text { Solutio } \\
\mathrm{n}\end{array}$ & \multicolumn{2}{|c|}{ Pseudorange observation equations } & $\begin{array}{l}\text { Ionospheric correction } \\
\text { applied to carrier phase }\end{array}$ \\
\hline 1 & \multicolumn{2}{|c|}{$P_{1}^{j}=\rho^{j}+c(d T)$} & none \\
\hline & XYZ & ionosphere & \\
\hline 2 & $P_{1}^{j}=\rho^{j}+c(d T)$ & $I_{R}=\left[\left(P_{2}-P_{1}\right) \cdot \frac{f_{2}^{2}}{f_{1}^{2}-f_{2}^{2}}\right] / M(e)$ & $\Phi_{i c}=\Phi_{i}+M(e) \gamma_{i} I_{R} f_{i} / c$ \\
\hline 3 & \multicolumn{2}{|c|}{$\begin{array}{c}P_{i}=\rho+c(d T)+M(e) \gamma_{i} I_{R} \\
C_{1}=\rho+c(d T)+M(e) I_{R}\end{array}$} & $\Phi_{i c}=\Phi_{i}+M(e) \gamma_{m} I_{R} f_{i} / c$ \\
\hline \multirow[b]{2}{*}{4} & & XYZ regional filter & \multirow[b]{2}{*}{$\Phi_{i c}=\Phi_{i}+M(e) \gamma_{i} I_{R} f_{i} / c$} \\
\hline & $\begin{array}{c}P_{i}=\rho+c(d T)+M(e) \gamma_{i} I_{R} \\
C_{1}=\rho+c(d T)+M(e) I_{R}\end{array}$ & $X_{r}=X_{m 0}-\left(X_{f 0}-X_{f t}\right)$ & \\
\hline \multirow{4}{*}{5} & \multirow{4}{*}{$P_{i}=\rho+c(d T)+M(e) \gamma_{i} I_{R}$} & XYZ regional filter & \multirow{4}{*}{$\Phi_{i c}=\Phi_{i}+M(e) \gamma_{i} I_{R} f_{i} / c$} \\
\hline & & $X_{r}=X_{m 0}-\left(X_{f 0}-X_{f t}\right)$ & \\
\hline & & XYZ sidereal filter & \\
\hline & & $X_{s}=X_{r 0}-\left(X_{r p}-X_{m t}\right)$ & \\
\hline
\end{tabular}

\section{Test dataset and results}

To study the GASP software positioning accuracy over medium and long baselines, data from a set of 10 International GNSS Service (IGS) sites in California has been processed. Table 2 and Fig. 1 show the processed stations and baselines. To avoid the possible effect of day boundaries, data has been analysed for the middle 21 hours of the tested days making a total of 2520 epochs per day (at $30 \mathrm{~s}$ intervals). Here we present data for day 66 of year 2007, which are typical of data spanning years 2007-2011 that we tested.

Table 2 Baselines processed from the test dataset (day 66, 2007). 


\begin{tabular}{|c|c|c|}
\hline $\begin{array}{c}\text { Base } \\
\text { station }\end{array}$ & $\begin{array}{c}\text { Rover } \\
\text { station }\end{array}$ & $\begin{array}{c}\text { Approximate } \\
\text { baseline } \\
\text { length }(\mathrm{km})\end{array}$ \\
\hline bran & leep & 7 \\
\hline uclp & leep & 13 \\
\hline uclp & bran & 19 \\
\hline leep & csn1 & 23 \\
\hline holp & leep & 27 \\
\hline azu1 & bran & 35 \\
\hline azu1 & leep & 39 \\
\hline bran & torp & 44 \\
\hline csn1 & holp & 50 \\
\hline csn1 & azu1 & 60 \\
\hline azu1 & crfp & 75 \\
\hline crfp & holp & 99 \\
\hline crfp & leep & 113 \\
\hline uclp & crfp & 123 \\
\hline csn1 & crfp & 133 \\
\hline gold & crfp & 155 \\
\hline pin1 & holp & 160 \\
\hline gold & leep & 198 \\
\hline
\end{tabular}

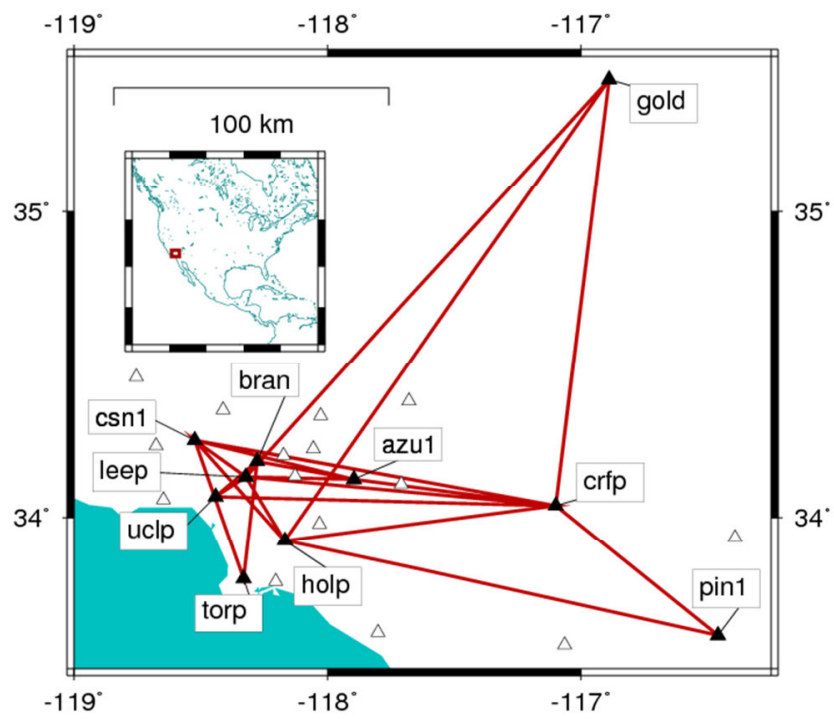

Fig. 1 IGS stations used in this study (named), and processed baselines (red lines).

Three main statistics have been computed to study the effectiveness of each of the processing strategies described above. The first is the Median Absolute Deviation (MAD) of the final estimated coordinates around the "true" position. MAD is used rather than the root mean square deviation, because the latter is unduly 
sensitive to large outliers. The second statistic is the percentage of epochs for which "good" results are obtained, defined as those which give 3-D positioning accuracy better than $10 \mathrm{~cm}$. The third is the percentage of the epochs for which the GASP software reports ambiguity resolution success, i.e. those for which the AFV is greater than the threshold value 0.9. For the position comparison, the 'true' station position coordinates have been taken to be those published on the Scripps Orbit and Permanent Array Center (SOPAC) website. The solution methods in Table 1 have been examined to decide which will give the best positions and ambiguity resolution success rates. Fig. 2 shows the results of using these methods on the test dataset.

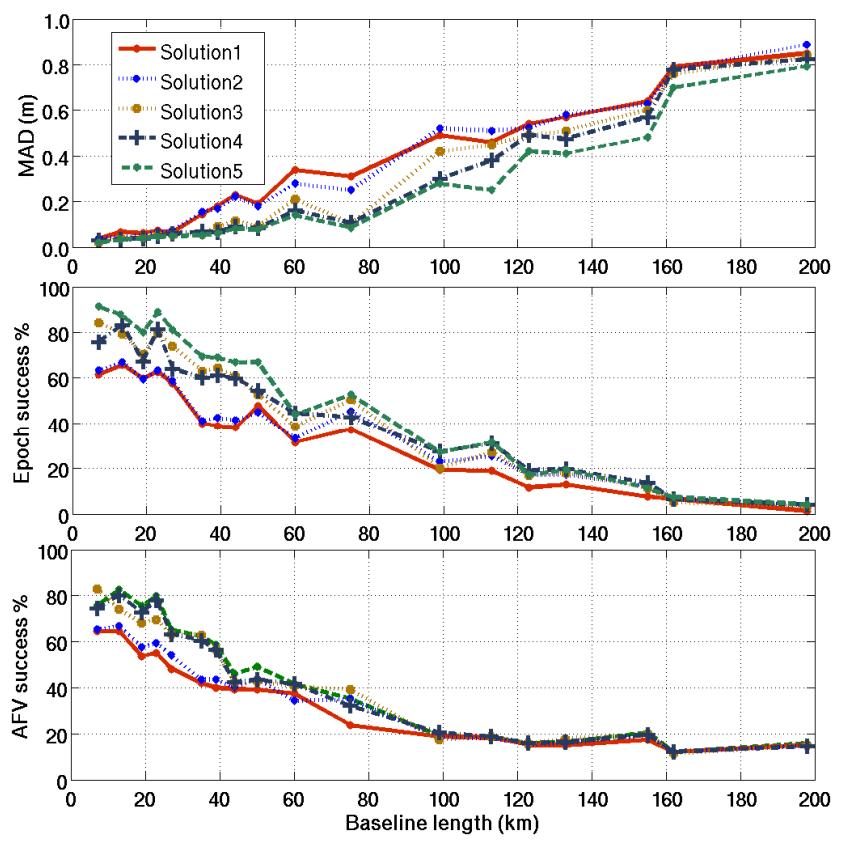

Fig. 2 Success statistics of the five tested methods as a function of baseline length.

Fig. 2 indicates, for most baselines, a progressive improvement in positioning as the complexity of the method increases from the basic solution 1 to solution 5 . For short baselines less than $25 \mathrm{~km}$, this improvement is not particularly noticeable in the MAD statistic because more than half of the epochs are within $10 \mathrm{~cm}$ of the true position for all solutions, but it is visible in the other measures of success. Using the geometry free observable to generate a local ionospheric model (solution 2) improves the results from the double differenced code solution (solution 1); as might be expected, this improvement is small for the short baselines for which double-differencing effectively removes the ionospheric error in the carrier phase solution, but more appreciable for baselines in the range $50-120 \mathrm{~km}$. In contrast, the undifferenced pseudorange trial coordinate solution (solution 3) gives significantly better final carrier phase positioning results than the double differenced pseudorange trial coordinate solution (solution 2), even though both methods include a residual ionospheric model estimate. We attribute this to several factors. First, the trial coordinates are less noisy than those of solution 1 or solution 2 , and so there is a greater chance of them falling within the range necessary for a successful solution. Second, the residual ionospheric model applied to the carrier phases is more precise and robust because it consists of a single zenith delay derived from dual-frequency pseudorange data of all satellites rather than individual values for each satellite derived from their dual-frequency code pseudoranges, and so there is greater redundancy in the observations. 
It can be also concluded from Fig. 2 that applying regional and sidereal filters to the trial coordinates (solutions 4 and 5) has a positive effect on the GASP carrier phase positioning results. In the case of regional filtering (solution 4), this improvement is very small except for the MAD of the final coordinates of baselines around 100-120 km. For sidereal filtering the improvement in MAD and epoch success rate is more pervasive, but this technique can only be applied when reliable data from the preceding day is available and for antennas which have moved sub-decimetre amounts in relation to nearby multipath reflectors over this time.

Fig. 3 shows a comparison between the solution 2 and 3 trial positions and the true station coordinates for a $60 \mathrm{~km}$ baseline. The improvement seen in the solution 3 trial positions may be partly because multipath and other site-specific errors are magnified in the case of a double-differenced solution as two stations are involved. Also, more data are available for use in the undifferenced solution than in the double-differenced solution, as there is no need to restrict the observations to shared satellites only.

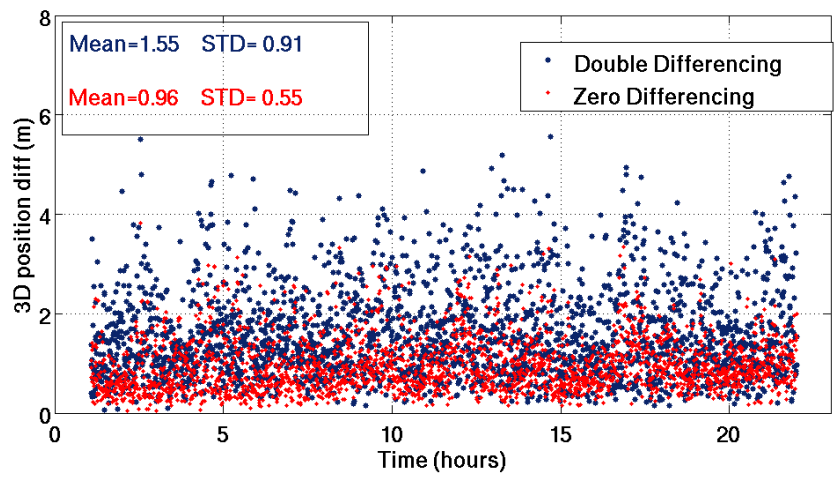

Fig. 3 Undifferenced and double-differenced pseudorange solution deviations from the true position, for a $60 \mathrm{~km}$ baseline (CSN1 - AZU1),

\section{Applying Kalman filtering to the ambiguity function inputs}

The ionosphere is not expected to change rapidly from epoch to epoch (Enge and Misra 1999); therefore it is possible to smooth the code-based residual ionospheric delay model values by combining results over time. A good way to do this is to employ a Kalman filter, which provides an efficient estimation of a dynamic system state from a series of noisy measurements (Kalman 1960; Kalman and Bucy 1961; Maybeck 1979). The Kalman filter employs a dynamic model, usually represented by a transition matrix, to predict the state vector and its covariance, and an observation model to correct the predicted state, and has found widespread usage in GPS applications.

In our case, the state vector of unknown parameters consists of the rover position and velocity, receiver clock correction, and zenith ionospheric delay. Although we include position, velocity and clock parameters, in the results presented we assign very large process noise to them so that they effectively remain independent at each epoch. For the zenith ionospheric delay, we assign process noise of $0.001 \mathrm{~m}^{2}$ which we have 
experimentally found to represent a suitable balance between over- and under-constraint. The input 'observations' to the Kalman filter are the results (rover position, receiver clock, and local zenith ionospheric delay correction, and their a posteriori full variance-covariance matrix scaled by the variance of unit weight) from the single-epoch undifferenced pseudorange solution (solution 3).

The position and zenith ionospheric delay outputs of the Kalman filter are used as the moving antenna initial position coordinates and to correct the GPS phase measurements respectively, in the GASP software ambiguity function. Fig. 4 shows the effect of applying the Kalman filter to obtain solution 3KF, compared with the previous (unfiltered) solution 3 results. Improvement in the MAD is seen for all baselines longer than $40 \mathrm{~km}$, and there are improvements in the epoch success rate for the majority of short- and medium-length baselines. The net effect is that single-epoch positioning with a MAD of less than $20 \mathrm{~cm}$ becomes possible up to baseline lengths of around $120 \mathrm{~km}$, which without the Kalman-filtered ionosphere was achieved only for baselines shorter than around $80 \mathrm{~km}$.
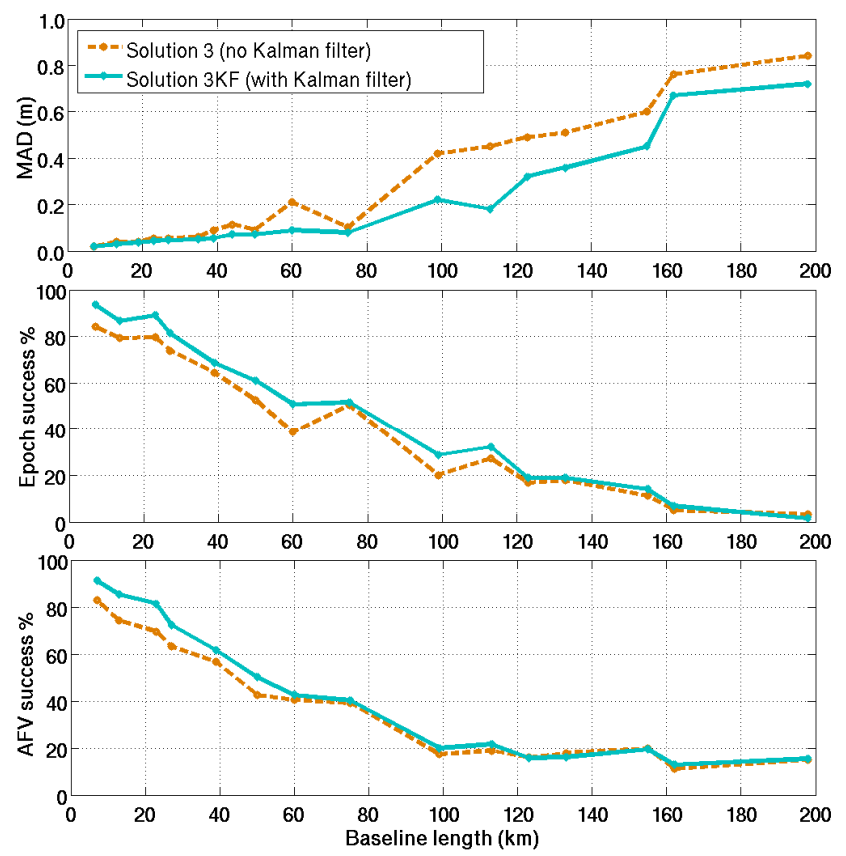

Fig. 4 Success metrics of the solution 3 results, with and without Kalman filtering.

In some situations, e.g. monitoring of decimetre-level or smaller movements, sufficiently accurate seed coordinates will be available for the rover site without the need for a pseudorange-based position solution. In such cases a local ionospheric model may still prove useful in the ambiguity search. To test this, we reprocessed the above solutions 1,3 and $3 \mathrm{KF}$ using fixed precise IGS site coordinates as the seed positions input to the ambiguity search in place of the variable pseudorange-derived ones used previously. Results are shown in Fig. 5. Barring minor differences caused by outlier rejection, the MAD of final coordinates does not vary with the introduction of the local ionospheric model. For baselines shorter than $80 \mathrm{~km}$ or longer than $150 \mathrm{~km}$ the MAD is similar to that seen in the variable-seed solution $3 \mathrm{KF}$, although for the longer baselines the number of final 
accepted solutions is small. For medium-length baselines of 100-140 km the MAD of the variable-seed solution $3 \mathrm{KF}$ is somewhat better than the fixed-seed $3 \mathrm{KFa}$, suggesting that the variable seed positions are partially able to absorb any deficiencies in the IGS or local ionospheric models resulting in an ionospheric correction that is more compatible with the carrier phase data. However, at all baseline lengths in the fixed-seed solutions we see a consistent improvement in the percentages of 'good' epochs and those passing the AFV threshold test, when going from the IGS ionospheric model to the locally-augmented ionospheric model whether epoch-by-epoch or with Kalman filtering. In comparison with Figs. 2 and 4, the success rate metrics are most similar to solution $3 \mathrm{KF}$. This suggests that the majority of the improvement seen in solution $3 \mathrm{KF}$ compared with the original solution 1 is the result of improved seed coordinates combined with more compatible local ionospheric corrections, with the latter also being a significant factor affecting the availability of solutions.

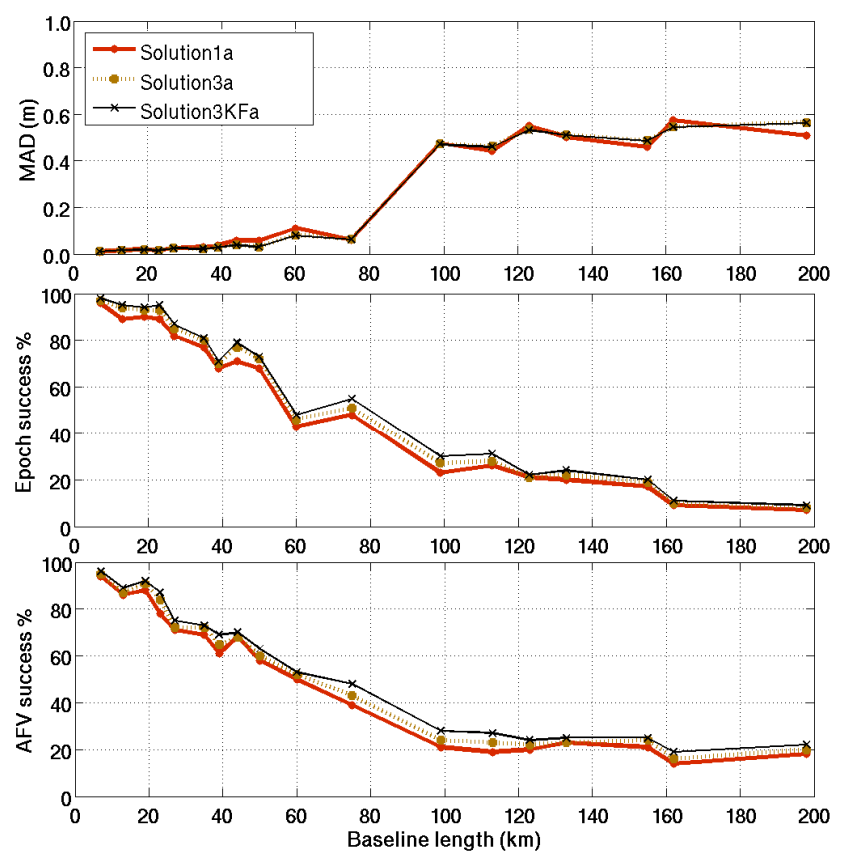

Fig. 5 Success metrics of ionospheric solutions 1, 3 and 3KF, with accurate seed position coordinates used in place of those obtained in the corresponding solutions described above.

\section{Conclusions}

Inclusion of a pseudorange-based estimate of local residual ionospheric delay, to refine the approximate rover antenna position and correct the carrier phase measurements, results in improved single-epoch GPS carrier phase ambiguity resolution and relative positioning for baselines longer than $30 \mathrm{~km}$. Using an undifferenced pseudorange solution to obtain the seed position gives better results than using a double differenced solution, as noise is reduced and more satellites are available to be used. More significant improvement arises from parameterising the local ionospheric model as a zenith delay rather than using satellite-specific terms, because the single zenith parameter is more precise and robust. In this way, single-epoch position accuracy 
corresponding to a median absolute deviation (MAD) of better than $20 \mathrm{~cm}$ can be achieved for baselines up to $80 \mathrm{~km}$.

Applying regional and sidereal filters to the undifferenced pseudorange trial position solution improves the final positioning results by a small amount, but does not increase the baseline length for which a MAD of better than $20 \mathrm{~cm}$ can be achieved. However, sidereal filtering has drawbacks as it is only available in the case of a stationary or near-stationary rover antenna, where the GPS errors have sidereal repeat patterns. It also requires the availability of data for at least one sidereal day before the processing epoch.

Applying a Kalman filter to smooth the estimates of ionospheric delay reduces the noise level on the local ionospheric model, which improves the quality of the corrected GPS phase measurements and hence the availability and quality of single-epoch position solutions. Following the application of this technique, singleepoch positioning with a MAD less than $20 \mathrm{~cm}$ becomes possible for baselines up to $120 \mathrm{~km}$. At this performance level, single-epoch positioning becomes a viable technique for monitoring at regional scales of larger civil engineering structural motions such as wind and traffic loading, and ground deformations such as landslips and earthquakes. For these and other applications, our technique may be a useful alternative to conventional network real time kinematic positioning (Leandro et al. 2011), because infrastructure and subscription costs are reduced. Furthermore, in treating each epoch independently the method is less sensitive to communications network outages, and the frequency of output positions may be tuned to the needs of the application rather than to the performance of cycle slip detection algorithms.

\section{References}

Corbett, S. and P. Cross (1995). GPS single epoch ambiguity resolution. Survey Review 33(257): 149-160.

Counselman, C. and S. Gourevitch (1981). Miniature Interferometer Terminals for Earth Surveying: Ambiguity and Multipath with Global Positioning System. IEEE Transactions on Geosciences and Remote Sensing 19(4): 244-252.

Enge, P. and P. Misra (1999). Special issue on global positioning system. Proceedings of the IEEE 87(1): 3-15.

Kalman, R. E. (1960). A New Approach to Linear Filtering and Prediction Problems. Transaction of the ASME, Journal of Basic Engineering 82: 34-45.

Kalman, R. E. and R. Bucy (1961). New results in Linear Filtering and Prediction Theory. Journal of Basic Engineering 83D: $95-108$.

Kim, D. and R. B. Langley (2000). GPS ambiguity resolution and validation: methodologies, trends and issues. Proceedings of the $7^{\text {th }}$ GNSS Workshop - International Symposium on GPS/GNSS, Seoul, Korea 30: 2.12.

Kouba, J. (2009). A guide to using International GNSS Service (IGS) products. $\underline{\text { Available at }}$ http://www.igs.org/components/usage.html.

Leandro, R., et al. (2011). RTX Positioning: The Next Generation of cm-accurate Real-Time GNSS Positioning. Proc ION ITM-2011, Institute of Navigation, San Diego, Califormia, pp. 1460-1475.

Leick, A. (2004). GPS Satellite Surveying. New York, John Wiley \& Sons Inc. 
Lejeune, S., G. Wautelet and R. Warnant (2012). Ionospheric effects on relative positioning within a dense GPS network. GPS Solutions 16(1): 105-116.

Mader, G. L. (1992). Rapid static and kinematic global positioning system solutions using the ambiguity function technique. Journal of Geophysical Research 97(B3): 3271-3283.

Maybeck, P. (1979). Stochastic Models, Estimation, and Control. New York, Academic Press, Inc.

Niell, A. E. (1996). Global Mapping Functions for the Atmosphere Delay at Radio Wavelengths. Journal of Geophysical Research 101(No.B2): 3227-3246.

Ragheb, A., P.J. Clarke, P.J. and S.J. Edwards (2007). GPS sidereal filtering: coordinate- and carrier-phaselevel strategies. Journal of Geodesy 81(5): 325-335.

Saastamoinen, J. (1972). Contribution to the Theory of Atmospheric Refraction. Journal of Geodesy 46(3): pp.279-298.

Schaer, S., W. Gurtner and J. Feltens (1998). IONEX: The IONosphere map eXchange Format Version 1. Available at http://www.igs.org/igscb/data/format/ionex1.pdf.

Schmid, R., M. Rothacher, D. Thaller and P. Steigenberger (2005). Absolute phase center corrections of satellite and receiver antennas. GPS Solutions 9(4): 283-293.

\section{Author Biographies}

Mohammed Assiadi has recently completed a PhD in GNSS geodesy at Newcastle University, and now works for Scopus Surveys based in Aberdeen, UK.

Stuart Edwards is Senior Lecturer in Geomatics at Newcastle University, where he has carried out research in GNSS geodesy and deformation monitoring for the last 19 years. Prior to that he was a chartered land surveyor for British Coal. His other research interests include the effects of tropospheric water vapour on GNSS and satellite radar altimeters.

Peter Clarke is Professor of Geophysical Geodesy at Newcastle University. His research interests include GNSS positioning, reference frames, surface mass loading of the earth, deformation throughout the seismic cycle and over post-glacial timescales, seal level change, and the effects of tropospheric water vapour on GNSS and InSAR. 